\title{
INEQUALITIES FOR SOME MAXIMAL FUNCTIONS. II
}

\author{
M. COWLING AND G. MAUCERI
}

\begin{abstract}
Let $S$ be a smooth compact hypersurface in $\mathbf{R}^{n}$, and let $\mu$ be a measure on $S$, absolutely continuous with respect to surface measure. For $t$ in $\mathbf{R}^{+}, \mu_{t}$ denotes the dilate of $\mu$ by $t$, normalised to have the same total variation as $\mu$ : for $f$ in $S\left(\mathbf{R}^{n}\right), \mu^{\#} f$ denotes the maximal function $\sup _{t>0}\left|\mu_{t} * f\right|$. We seek conditions on $\mu$ which guarantee that the $a$ priori estimate

$$
\left\|\mu^{\#} f\right\|_{p} \leq C\|f\|_{p}, \quad f \in S\left(\mathbf{R}^{n}\right),
$$

holds; this estimate entails that the sublinear operator $\mu^{\#}$ extends to a bounded operator on the Lebesgue space $L^{p}\left(\mathbf{R}^{n}\right)$. Our methods generalise E. M. Stein's treatment of the "spherical maximal function" [5]: a study of "Riesz operators", $g$-functions, and analytic families of measures reduces the problem to that of obtaining decay estimates for the Fourier transform of $\mu$. These depend on the geometry of $S$ and the relation between $\mu$ and surface measure on $S$. In particular, we find that there are natural geometric maximal operators limited on $L^{p}\left(\mathbf{R}^{n}\right)$ if and only if $p \in(q, \infty) ; q$ is some number in $(1, \infty)$, and may be greater than 2 . This answers a question of $S$. Wainger posed by Stein [6].
\end{abstract}

0. Notation and contents. The following notation will be used: the Fourier transform $\hat{f}$ of a function $f$ on $\mathbf{R}^{n}$ is given by

$$
\hat{f}(\xi)=\int_{\mathbf{R}^{n}} d x f(x) e^{-2 \pi i \xi \cdot x}, \quad \xi \in \mathbf{R}^{n} .
$$

By $B(x, r)$ we mean the open ball of radius $r$ and centre $x$. The unit sphere in $\mathbf{R}^{n}$ is $S^{n-1}$; we often use polar coordinates $\rho$ and $\sigma$, with $\rho$ in $[0, \infty)$ and $\sigma$ in $S^{n-1}$. By expressions like $C, C_{p}, C_{k, R}$, etc., we denote various constants, which may vary from line to line, and which depend on $p, k$ and $R$, and so on. These constants will normally also depend on other parameters, like $n$, the dimension of the ambient space, but we do not mention this dependence explicitly.

Given $f$ in $S\left(\mathbf{R}^{n}\right)$, we denote by $\delta_{t} f$ and $D_{t} f$ the dilates:

$$
\begin{array}{ll}
\delta_{t} f(x)=t^{-n} f\left(t^{-1} x\right), & t \in \mathbf{R}^{+}, x \in \mathbf{R}^{n}, \\
D_{t} f(x)=f(t x), & t \in \mathbf{R}^{+}, x \in \mathbf{R}^{n} .
\end{array}
$$

These are related by the Fourier transform $\left(\delta_{t} f\right)^{-}=D_{t} \hat{f}$. For a distribution $\phi$ in $S^{\prime}\left(\mathbf{R}^{n}\right)$, define $\delta_{t} \phi$ and $D_{t} \phi$ by duality:

$$
\begin{array}{ll}
\left\langle\delta_{t} \phi, f\right\rangle=\left\langle\phi, D_{t} f\right\rangle, & t \in \mathbf{R}^{+}, f \in S\left(\mathbf{R}^{n}\right), \\
\left\langle D_{t} \phi, f\right\rangle=\left\langle\phi, \delta_{t} f\right\rangle, & t \in \mathbf{R}^{+}, f \in S\left(\mathbf{R}^{n}\right) .
\end{array}
$$

Received by the editors August 13, 1985.

1980 Mathematics Subject Classification. Primary 42B25; Secondary 42B10.

Key words and phrases. Maximal function, hypersurface, Fourier transform. 
For $f$ in $S$ and $\phi$ in $S^{\prime}$, the maximal function $\phi^{\#} f$ is defined thus:

$$
\phi^{\#} f=\sup \left\{\left|\delta_{t} \phi * f\right|: t \in \mathbf{R}^{+}\right\} .
$$

In $\S \S 1-3$ of this paper, we treat the theory of maximal functions associated to measures supported by surfaces; roughly speaking, the three sections contain the cases $p=2,1<p<2$, and $p>2$. $\S 4$ contains a family of examples; the estimation of the Fourier transforms in $\S 4$ requires many applications of van der Corput's lemma. We have grouped these together in an Appendix.

This work was announced in 1980 by the first-named author [1]. Related work has been presented by A. Greenleaf $[\mathbf{3}]$, and can be found in the recent preprint of C. D. Sogge and E. M. Stein [4], which arrived while this paper was being typed.

1. The $L^{2}$-theory. In this section, we prove that the maximal operator $\phi^{\#}$ is $L^{2}$-bounded for a compactly supported distribution $\phi$ whose Fourier transform vanishes fast enough at infinity. The proof hinges on a study of "Riesz operators", introduced below, and a $g$-function argument. In particular, Lemma 2 examines the effect of Riesz operators on Fourier transform decay and Lemma 3 studies their composition. The techniques of Lemma 2 come from the theory of fractional differentiation, and were well known to the experts. Lemma 3 involves a real variable argument which is simpler than some other approaches, which superficially invoke properties of Bessel functions (e.g. Stein [5]). Finally, Theorem 1 is based on the work of Stein (see also Stein and Wainger [7]).

Our first result, a preliminary lemma, is standard.

LEMMA 1.1. Let $\phi$ be a distribution supported in $B(0, R)$. Suppose that, for some $\alpha$ in $\mathbf{R}$,

$$
|\hat{\phi}(\rho \sigma)| \leq(1+\rho)^{\alpha}, \quad \sigma \in S^{n-1}, \rho \in \mathbf{R}^{+} .
$$

Then $\hat{\phi}$ is $C^{\infty}$, and further, for all $k$ in $\mathbf{N}$, there are constants $C_{k, R}$ such that

$$
\left|\partial^{k} / \partial \rho^{k} \hat{\phi}(\rho \sigma)\right| \leq C_{k, R}(1+\rho)^{\alpha}, \quad \sigma \in S^{n-1}, \rho \in \mathbf{R}^{+} .
$$

ProOF. It is well known that $\hat{\phi}$ is real analytic, so $\hat{\phi}$ is certainly $C^{\infty}$. Next, if $h$ in $S(\mathbf{R})$ agrees with the function $t \rightarrow(-2 \pi i t)^{k}$ on a neighborhood of $[-R, R]$, then

$$
\partial^{k} / \partial \rho^{k} \hat{\phi}(\rho \sigma)=\int_{\mathbf{R}} d r \hat{h}(\rho-r) \hat{\phi}(r \sigma)
$$

from which the lemma follows.

Now we define the Riesz operators: later we study their properties. For $a, z$ in C, with $\operatorname{Re}(a)>0$ and $f$ in $S\left(\mathbf{R}^{n}\right)$, we define $R_{a, z} f$ as follows: if $\operatorname{Re}(z)>0$, then

$$
R_{a, z} f(x)=2 \Gamma(z)^{-1} \int_{0}^{1} d t t^{a-1}\left(1-t^{2}\right)^{z-1} \delta_{t} f(x), \quad x \in \mathbf{R}^{n}
$$

This integral converges absolutely, and $R_{a, z} f$ is integrable and rapidly decreasing. For fixed $a$ and $x, z \rightarrow R_{a, z} f(x)$ continues analytically into C. Indeed, if $x=0$, $\delta_{t} f(x)$ is constant, and the claim is clear. Otherwise, $t \rightarrow \delta_{t} f(x)$ vanishes rapidly as $t \rightarrow 0+$ and is $C^{\infty}$, so admits a Taylor expansion about 1 of arbitrary order. 
Then for any $M$ in $\mathbf{N}$,

$$
\begin{aligned}
& 2 \Gamma(z)^{-1} \int_{0}^{1} d t t^{a-1}\left(1-t^{2}\right)^{z-1} \delta_{t} f(x) \\
& \quad=2 \Gamma(z)^{-1} \int_{0}^{1} d t t^{a-1}\left(1-t^{2}\right)^{z-1}\left(\sum_{m=0}^{M-1} a_{m}(1-t)^{m}+R_{M}(t)\right),
\end{aligned}
$$

where the remainder $R_{M}(t)$ is bounded and satisfies $R_{M}(t)=O\left((1-t)^{M}\right)$. Our integral breaks into the sum of $M B$-like integrals, whose poles are annihilated by the factor $\Gamma(z)^{-1}$, and an integral involving the remainder which continues into the region $\operatorname{Re}(z)>-M$. The analytic continuation follows by the arbitrary size of $M$.

By this same argument, it is clear that $R_{a, z} f$ is $C^{\infty}$ away from 0 and is differentiable at 0 to any preassigned order, provided that $a$ is large enough.

Note that $R_{a, 0} f=f$.

Clearly, if $\operatorname{Re}(z)>0$, then

$$
\left(R_{a, z} f\right)^{-}(\xi)=2 \Gamma(z)^{-1} \int_{0}^{1} d t t^{a-1}\left(1-t^{2}\right)^{z-1} D_{t} \hat{f}(\xi), \quad \xi \in \mathbf{R}^{n}
$$

this continues analytically in $z$ for similar reasons.

If $\phi$ is a compactly supported distribution on $\mathbf{R}^{n}$, then we may define $R_{a, z} \phi$ by duality: for $f$ in $S\left(\mathbf{R}^{n}\right)$,

$$
\left\langle R_{a, z} \phi, f\right\rangle=2 \Gamma(z)^{-1} \int_{0}^{1} d t t^{a-1}\left(1-t^{2}\right)^{z-1}\left\langle\phi, D_{t} f\right\rangle
$$

or equivalently,

$$
\left\langle R_{a, z} \phi, \hat{f}\right\rangle=\left\langle\hat{\phi}, R_{a, z} f\right\rangle .
$$

Our next lemma shows that, if $\operatorname{Re}(z)<0, R_{a, z}$ is like a pseudodifferential operator of order $z$.

LEMMA 1.2. Suppose that $\phi$ in $S^{\prime}\left(\mathbf{R}^{n}\right)$ is supported in $B(0, R)$, and that for some $\alpha$ in $\mathbf{R}$,

$$
|\hat{\phi}(\rho \sigma)| \leq C(1+\rho)^{\alpha}, \quad \sigma \in S^{n-1}, \rho \in \mathbf{R}^{+} .
$$

Then $R_{a, z} \phi$ is in $S^{\prime}\left(\mathbf{R}^{n}\right)$ and is supported in $B(0, R)$. Further, if $\operatorname{Re}(a)+\alpha>0$ and if $\varepsilon>0$, then

$$
\left|\left(R_{a, z} \phi\right)^{\uparrow}(\rho \sigma)\right| \leq C(1+\rho)^{\alpha+m}, \quad \sigma \in S^{n-1}, \rho \in \mathbf{R}^{+},
$$

where $m=\max (0,-\operatorname{Re}(z))+\varepsilon$.

PROOF. The support of $R_{a, z} \phi$ is obviously contained in $B(0, R)$. Further, if $\sigma \in S^{n-1}$ and $\rho \geq 1$, then

$$
\begin{aligned}
\left(R_{a, z} \phi\right)^{\wedge}(\rho \sigma)=2 \Gamma(z)^{-1}\left\{\int_{0}^{1 / 2 \rho} d t t^{a-1}\left(1-t^{2}\right)^{z-1} \hat{\phi}(t \rho \sigma)\right. & \\
& \left.+\int_{1 / 2 \rho}^{1} d t t^{a-1}\left(1-t^{2}\right)^{z-1} \hat{\phi}(t \rho \sigma)\right\} .
\end{aligned}
$$


The first integral is bounded by

$$
C_{z, \alpha} \int_{0}^{1 / 2 \rho} d t\left|t^{a-1}\right|=O\left(\rho^{-\operatorname{Re}(a)}\right)
$$

the second integral is easy if $\operatorname{Re}(z)>0$ and is treated by complex interpolation otherwise. If $\operatorname{Re}(z) \geq \varepsilon>0$, then

$$
\begin{aligned}
& \left|\int_{1 / 2 \rho}^{1} d t t^{a-1}\left(1-t^{2}\right)^{z-1} \hat{\phi}(t \rho \sigma)\right| \\
& \quad \leq C \int_{1 / 2 \rho}^{1} d t\left|t^{a-1}\right|\left(1-t^{2}\right)^{\varepsilon-1}(t \rho)^{\alpha} \leq C_{a, \varepsilon} \rho^{\alpha} .
\end{aligned}
$$

Moreover, by the Mean Value Theorem

$$
\hat{\phi}(t \rho \sigma)=\hat{\phi}(\rho \sigma)-\left(1-t^{2}\right) \theta(t)
$$

where $(1+t) \theta(t)=\partial / \partial s \hat{\phi}(s \rho \sigma)$, for some $s$ (depending measurably on $t$ ) between $t$ and 1 . Hence, if $\operatorname{Re}(z) \geq \varepsilon-1$, then because $|\theta(t)| \leq C \rho(t \rho)^{\alpha}$ by Lemma 1 , we have

$$
\begin{aligned}
& \left|\int_{1 / 2 \rho}^{1} d t t^{a-1}\left(1-t^{2}\right)^{z-1} \hat{\phi}(t \rho \sigma)\right| \\
& \quad \leq\left|\int_{1 / 2 \rho}^{1} d t t^{a-1}\left(1-t^{2}\right)^{z-1}\right||\hat{\phi}(\rho \sigma)|+\left|\int_{1 / 2 \rho}^{1} d t t^{a-1}\left(1-t^{2}\right)^{z} \theta(t)\right| \\
& \quad \leq C_{a, z}(1+\rho)^{\alpha}+C_{\alpha} \int_{1 / 2 \rho}^{1} d t\left|t^{a-1}\right|\left(1-t^{2}\right)^{\varepsilon-1} \rho(t \rho)^{\alpha} \\
& \quad \leq C_{a, z, \alpha}(1+\rho)^{\alpha+1} .
\end{aligned}
$$

The constants involved grow at most exponentially in $|z|$, so complex interpolation shows that if $-1<\operatorname{Re}(z) \leq 0$, then

$$
\left|R_{a, z} \hat{\phi}(\xi)\right| \leq C(1+\rho)^{\alpha-\operatorname{Re}(z)+\varepsilon}
$$

(See e.g. E. M. Stein and G. Weiss [8] for a discussion of the complex interpolation method.)

In order to treat $z$ with $\operatorname{Re}(z) \leq-1$, we note that (1.1) is just a low order Taylor expansion of $t \rightarrow \hat{\phi}(t \rho \sigma)$ about $t=1$ : by using the expression

$\hat{\phi}(t \rho \sigma)=\sum_{m=0}^{m-1}(m !)^{-1}(t-1)^{m}\left[\partial^{m} / \partial t^{m} \hat{\phi}(t \rho \sigma)\right]_{t=1}+(M !)^{-1}(t-1)^{M} \partial^{M} / \partial s^{M} \hat{\phi}(s \rho \sigma)$

for some $s$ between $t$ and 1 , we may show that, if $\operatorname{Re}(z) \geq \varepsilon-M-1$, then

$$
\left|\int_{1 / 2 \rho}^{1} d t t^{a-1}\left(1-t^{2}\right)^{z-1} \hat{\phi}(t \rho \sigma)\right| \leq C_{a, z, \alpha}(1+\rho)^{\alpha+M+1}
$$

and deduce the result of the lemma for $z$ with $\operatorname{Re}(z)>-M-1$ by complex interpolation. 
It may be of interest to note that, if $\hat{\phi}$ admits an asymptotic expansion like that of a Bessel function, then one can show that

$$
\left.\mid\left(R_{a, z} \phi\right)\right\urcorner(\rho \sigma) \mid \leq C(1+\rho)^{\alpha-\operatorname{Re}(z)+\varepsilon}
$$

for $\operatorname{Re}(z)>0$, provided that $\operatorname{Re}(a)$ is large enough. The Fourier transform of measures we consider later fall into this category.

Our third lemma gives a formula for the composition of two Riesz operators. Its form is one justification for the normalisation of $R_{a, z}$.

LEMMA 1.2. When $\operatorname{Re}(z), \operatorname{Re}(a-2 w)>0$, we have

$$
R_{a-2 w, w} R_{a, z}=R_{a-2 w, w+z}, \quad w, z \in \mathbf{C} .
$$

ProOF. We may assume that $\operatorname{Re}(z)$ and $\operatorname{Re}(w)$ are positive, and then obtain the general result by analytic continuation. For $\xi$ in $\mathbf{R}^{n}$ and $f$ in $S\left(\mathbf{R}^{n}\right)$,

$$
\begin{aligned}
& \left(R_{a-2 w, w}\left(R_{a, z} f\right)\right)^{\uparrow}(\xi) \\
& \quad=4 \Gamma(w)^{-1} \Gamma(z)^{-1} \int_{0}^{1} d s \int_{0}^{1} d t s^{a-2 w-1}\left(1-s^{2}\right)^{w-1} t^{a-1}\left(1-t^{2}\right)^{z-1} \hat{f}(s t \xi) .
\end{aligned}
$$

In order to evaluate this expression, we set

$$
\begin{aligned}
& u=s t, \quad v=\left(1-t^{2}\right)^{1 / 2}\left(1-s^{2} t^{2}\right)^{-1 / 2} \\
& g=g(u, v)=1+u^{2} v^{2}-v^{2}
\end{aligned}
$$

Then

$$
\begin{gathered}
1-t^{2}=v^{2}\left(1-u^{2}\right), \quad t=g^{1 / 2}, \\
s=u g^{-1 / 2}, \quad 1-s^{2}=\left(1-u^{2}\right)\left(1-v^{2}\right) g^{-1} .
\end{gathered}
$$

We have a bijection of $[0,1]^{2}$ onto $[0,1]^{2}$, and

$$
\begin{gathered}
\partial t / \partial u=\frac{1}{2} g^{-1 / 2} \partial g / \partial u, \quad \partial t / \partial v=\frac{1}{2} g^{-1 / 2} \partial g / \partial v \\
\partial s / \partial u=g^{-1 / 2}-\frac{1}{2} u g^{-3 / 2} \partial g / \partial u, \quad \partial s / \partial v=-\frac{1}{2} u g^{-3 / 2} \partial g / \partial v
\end{gathered}
$$

whence the Jacobian determinant can readily be evaluated:

$$
\left|\frac{\partial(s, t)}{\partial(u, v)}\right|=-\frac{1}{2} g^{-1} \frac{\partial g}{\partial v}=g^{-1}\left(1-u^{2}\right) v \text {. }
$$

Substituting, we find that

$$
\begin{aligned}
& \left(R_{a-2 w, w}\left(R_{a, z} f\right)\right)^{-}(\xi)=4 \Gamma(w)^{-1} \Gamma(z)^{-1} \\
& \quad \times \int_{0}^{1} d u \int_{0}^{1} d v u^{a-2 w-1}\left(1-u^{2}\right)^{w+z-1} v^{2 z-1}\left(1-v^{2}\right)^{w-1} \hat{f}(u \xi) \\
& =\left(R_{a-2 w, w+z} f\right)^{-}(\xi)
\end{aligned}
$$

because $\int_{0}^{1} d v v^{2 z-1}\left(1-v^{2}\right)^{w-1}=\frac{1}{2} B(w, z)$.

We conclude this section with our main $L^{2}$-boundedness theorem. 
THEOREM 1.4. Let $\phi$ be a compactly supported distribution on $\mathbf{R}^{n}$. If for some $\alpha$ in $\left(-\infty,-\frac{1}{2}\right)$ we have

$$
|\hat{\phi}(\rho \sigma)| \leq C(1+\rho)^{\alpha}, \quad \sigma \in S^{n-1}, \rho \in \mathbf{R}^{+},
$$

then the maximal operator satisfies the a priori inequality

$$
\left\|\phi^{\#} f\right\|_{2} \leq C\|f\|_{2}, \quad f \in S\left(\mathbf{R}^{n}\right) .
$$

Proof. Choose $\beta$ in $\mathbf{R}, h$ in $S\left(\mathbf{R}^{n}\right)$ and $\varepsilon$ in $\mathbf{R}^{+}$so that $\max (\alpha,-3 / 4)<\beta<$ $-1 / 2, \hat{h}(0)=\left(R_{2, \beta} \phi\right) \widehat{\uparrow}(0)$ and $0<\varepsilon<\beta-\alpha$. Then

$$
\left|\left[R_{2, \beta} \phi-h\right]^{\wedge}(t \sigma)\right| \leq C t, \quad \sigma \in S^{n-1}, t \in[0,1],
$$

and

$$
\left|\left[R_{2, \beta} \phi-h\right]^{\Upsilon}(t \sigma)\right| \leq C(1+t)^{-\varepsilon}, \quad \sigma \in S^{n-1}, t \in(1, \infty),
$$

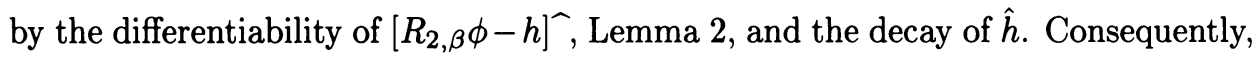

$$
\left\{\int_{0}^{\infty} d t t^{-1}\left|\left[R_{2, \beta} \phi-h\right]^{\Upsilon}(t \sigma)\right|^{2}\right\}^{1 / 2} \leq C, \quad \sigma \in S^{n-1} .
$$

By Lemma 3,

$$
\begin{aligned}
\delta_{s} \phi * f(x) & =\left(R_{2+2 \beta,-\beta}\left(R_{2, \beta} \delta_{s} \phi\right)\right) * f(x) \\
& =\left(R_{2+2 \beta,-\beta}\left(\delta_{s} R_{2, \beta} \phi\right)\right) * f(x),
\end{aligned}
$$

so that

$$
\begin{aligned}
\left|\delta_{s} \phi * f(x)\right|= & \left|\int_{0}^{1} d t t^{1+2 \beta}\left(1-t^{2}\right)^{-\beta-1}\left(\delta_{s t} R_{2, \beta} \phi\right) * f(x)\right| \\
\leq & \left\{\int_{0}^{1} d t t^{2+4 \beta}\left(1-t^{2}\right)^{-2 \beta-2}\right\}^{1 / 2} \\
& \times\left\{\int_{0}^{1} d t\left|\left(\delta_{s t} R_{2, \beta} \phi\right) * f(x)\right|^{2}\right\}^{1 / 2} \\
\leq & C_{\beta}\left\{s^{-1} \int_{0}^{s} d t\left|\left(\delta_{t} R_{2, \beta} \phi\right) * f(x)\right|^{2}\right\}^{1 / 2} \\
\leq & C_{\beta}\left\{s^{-1} \int_{0}^{s} d t\left|\left(\delta_{t}\left[R_{2, \beta} \phi-h\right]\right) * f(x)\right|^{2}\right\}^{1 / 2} \\
& +C_{\beta}\left\{s^{-1} \int_{0}^{s} d t\left|\left(\delta_{t} h\right) * f(x)\right|^{2}\right\}^{1 / 2} \\
\leq & C_{\beta}\left\{\int_{0}^{s} d t t^{-1}\left|\left(\delta_{t}\left[R_{2, \beta} \phi-h\right]\right) * f(x)\right|^{2}\right\}^{1 / 2} \\
& +C_{\beta}\left\{s^{-1} \int_{0}^{s} d t\left|h^{\#} f(x)\right|^{2}\right\}^{1 / 2} \\
\leq & C_{\beta}\left\{\int_{0}^{\infty} d t t^{-1}\left|\left(\delta_{t}\left[R_{2, \beta} \phi-h\right]\right) * f(x)\right|^{2}\right\}^{1 / 2}+C_{\beta} h^{\#} f(x) \\
= & C_{\beta} g(f)(x)+C_{\beta} h^{\#} f(x),
\end{aligned}
$$


say. This final expression is independent of $s$, so maximises $\phi^{\#} f(x)$. Because $h^{\#} f$ can be dominated by a multiple of the Hardy-Littlewood maximal function of $f$,

$$
\left\|h^{\#} f\right\|_{2} \leq C\|f\|_{2}, \quad f \in L^{2}\left(\mathbf{R}^{n}\right) .
$$

Thus, to finish the proof, it suffices to estimate $\|g(f)\|_{2}$.

By Plancherel's and Fubini's theorems, a change of variables, and (1.2),

$$
\begin{aligned}
\left\{\int_{\mathbf{R}^{n}} d x|g(f)(x)|^{2}\right\}^{1 / 2} & =\left\{\int_{\mathbf{R}^{n}} d \xi \int_{0}^{\infty} d t t^{-1}\left|\left[R_{2, \beta} \phi-h\right]^{-}(t \xi) \hat{f}(\xi)\right|^{2}\right\}^{1 / 2} \\
& =\left\{\int_{\mathbf{R}^{n}} d \xi \int_{0}^{\infty} d t t^{-1}\left|\left[R_{2, \beta} \phi-h\right]^{-}\left(t \xi^{\prime}\right) \hat{f}(\xi)\right|^{2}\right\}^{1 / 2} \\
& \leq C\|\hat{f}\|_{2}=C\|f\|_{2}, \quad f \in S\left(\mathbf{R}^{n}\right)
\end{aligned}
$$

(here $\xi=|\xi| \xi^{\prime}$ ), by (1.2).

We avoided using the Mellin transform techniques of our previous paper [2] in this section. The Mellin method expresses $\phi$ as an integral:

$$
\hat{\phi}(\rho \sigma)=\int_{\mathbf{R}} d \gamma c(\sigma, \gamma) \rho^{i \gamma},
$$

where $c(\sigma, \gamma)=(2 \pi)^{-1} \int_{\mathbf{R}^{+}} d \rho \rho^{-1-i \gamma} \hat{\phi}(\rho \sigma)$, and then one has (essentially)

$$
\left\|\phi^{\#} f\right\|_{2} \leq \int_{\mathbf{R}} d \gamma\|c(\cdot, \gamma)\|_{L^{\infty}}\|f\|_{2}
$$

(actually one works with $\phi-h$, with $h$ on $S\left(\mathbf{R}^{n}\right)$, as above). In order to obtain good estimates for the integral, one needs to have

$$
|\hat{\phi}(\rho \sigma)| \leq C(1+\rho)^{\alpha}, \quad \sigma \in S^{n-1},
$$

for some $\alpha$ in $\left(-\infty,-\frac{1}{2}\right)$, and also some oscillatory control, which can be verified in the examples involving surfaces. One also needs a stronger version of Lemma 2, involving oscillatory behaviour, in order to obtain $L^{p}$-estimates: this can be done, but is more involved. Finally, the Mellin approach requires uniformity in $\sigma$ which is more complicated than in the $g$-function approach. In fact, there are (rather unnatural) examples, which the $g$-function can handle, for which

$$
\int_{\mathbf{R}} d \gamma|c(\sigma, \gamma)| \leq C, \quad \sigma \in S^{n-1},
$$

but also $\int_{\mathbf{R}} d \gamma\|c(\cdot, \gamma)\|_{L^{\infty}}=\infty$, so that the Mellin technique fails where the $g$ function works.

Our closing remark on Theorem 1 is quite obvious: it applies to distributions $\phi$ which do not have compact support but which satisfy the conclusions of Lemma 1 .

2. $L^{p}$-estimates. $1<p<2$. Hereafter, we consider measures supported by curved surfaces. Let $T: \mathbf{R}^{n} \backslash\{0\} \rightarrow \mathbf{R}^{+}$be a positive Lipschitz function, homogeneous of degree 1 , and let $S$ be the level set:

$$
S=\left\{x \in \mathbf{R}^{n}: T(x)=1\right\} .
$$

Then $S$ is a Lipschitz surface, and $\nabla T$ is an outward normal vector to $S$. (Conversely, given a Lipschitz surface $S$, which intersects each ray from the origin 
in one point, we can define $T$ by the rule $T(x)=\lambda \Leftrightarrow \lambda^{-1} x \in S, \lambda \in \mathbf{R}^{+}$, $x \in \mathbf{R}^{n} \backslash\{0\}$.) We shall be interested in measures $\mu$ of the form $d \mu(x)=h(x) d S(x)$, where $h: \mathbf{R}^{n} \backslash\{0\} \rightarrow \mathbf{R}$ is a Borel function, homogeneous of degree 0 , and $d S(x)$ is the usual element of surface area.

By using Stein's complex interpolation theorem, we prove that if

$$
|\hat{\mu}(\rho \sigma)| \leq C(1+\rho)^{-\alpha}, \quad \sigma \in S^{n-1}, \rho \in \mathbf{R}^{+},
$$

for some $\alpha$ in $\left(\frac{1}{2},(n-1) / 2\right)$, then $\mu^{\#}$ is $L^{p}$-bounded for $p$ in $(1+1 / 2 \alpha, \infty)$. The method is an extension of that of Stein [5]: we show that $\left(R_{n, z} \mu\right)^{\#}$ is bounded on $L^{p}\left(\mathbf{R}^{n}\right)$ for all $p$ in $(1, \infty)$ if $\operatorname{Re}(z) \geq 1$, and that $\left(R_{n, z} \mu\right)^{\#}$ is $L^{2}$-bounded for $\operatorname{Re}(z)>\frac{1}{2}-\alpha$, then interpolate. The only difficulty lies in describing $R_{n, z} \mu$ for $\operatorname{Re}(z)>0$; this is tackled in Proposition 2.1. The main result, Theorem 2.2, follows.

We use the following notation:

$$
E(t)=\left\{x \in \mathbf{R}^{n}: T(x) \leq t\right\} \cup\{0\}, \quad t \in \mathbf{R}^{+} .
$$

PROPOSITION 2.1. If $\operatorname{Re}(z)>0$, then $R_{n, z} \mu$ "is" the function

$$
\left(R_{n, z} \mu\right)(x)= \begin{cases}{\left[1-T(x)^{2}\right]^{z-1}|\nabla T(x)| h(x),} & x \in E(1), \\ 0, & x \notin E(1) .\end{cases}
$$

ProOF. Observe that, if $\operatorname{Re}(z)>1$ and $g \in S\left(\mathbf{R}^{n}\right)$, then

$$
\begin{aligned}
\int_{E(1)} & d x g(x)\left[1-T(x)^{2}\right]^{z-1} \\
& =-\int_{E(1)} d g g(x) \int_{T(x)}^{1} d t\left(\left[1-t^{2}\right]^{z-1}\right)^{\prime} \\
& =-\int_{0}^{1} d t\left(\left[1-t^{2}\right]^{z-1}\right)^{\prime} \int_{E(t)} d x g(x) \\
& =-\int_{0}^{1} d t\left(\left[1-t^{2}\right]^{z-1}\right)^{\prime} t^{n} \int_{E(1)} d x g(t x) \\
& =\int_{0}^{1} d t\left[1-t^{2}\right]^{z-1}\left\{n t^{n-1} \int_{E(1)} d x g(t x)+t^{n} \int_{E(1)} d x x \cdot \nabla[g(t x)]\right\}
\end{aligned}
$$

by changing the order of integration and variables, and integrating by parts. Another integration by parts shows that

$$
\int_{E(1)} d x x \cdot \nabla[g(t x)]=\int_{S} d S(x) g(t x) x \cdot n(x)-n \int_{E(1)} d x g(t x)
$$

where $n(x)$ is the outward unit normal to $E(1)$. Thus

$$
\begin{aligned}
\int_{E(1)} & d x g(x)\left[1-T(x)^{2}\right]^{z-1} \\
= & \int_{0}^{1} d t\left[1-t^{2}\right]^{z-1} t^{n-1} \int_{S} d S(x) g(t x)(x \cdot n(x)) .
\end{aligned}
$$

By approximating by smooth functions, we see that this formula is still valid for continuous and for Borel measurable $g$. 
Since $T$ is homogeneous of degree 1, Euler's differential equation for homogeneous functions implies that, if $T(x)=1$,

$$
\|\nabla T(x)\|(x \cdot n(x))=x \cdot \nabla T(x)=T(x)=1 .
$$

Consequently, putting $g(x)=f(x)|\nabla T(x)| h(x)$ in (2.1), we find that

$$
\begin{aligned}
\int_{E(1)} & d x f(x)\left[1-T(x)^{2}\right]^{z-1}|\nabla T(x)| h(x) \\
= & \int_{0}^{1} d t t^{n-1}\left[1-t^{2}\right]^{z-1} \int_{S} d x(x) f(t x) h(x) \\
= & \int_{0}^{1} d t t^{n-1}\left[1-t^{2}\right]^{z-1} \int_{\mathbf{R}^{n}} d \mu(x) D_{t} f(x),
\end{aligned}
$$

which proves our claim when $\operatorname{Re}(z)>1$. For other values of $z$, the result follows by analytic continuation.

THEOREM 2.2. If, for some $\alpha$ in $\left(\frac{1}{2},(n-1) / 2\right]$,

$$
|\hat{\mu}(\rho \sigma)| \leq C(1+\rho)^{-\alpha}, \quad \sigma \in S^{n-1}, \rho \in \mathbf{R}^{+},
$$

then the maximal operator $\mu^{\#}$ is $L^{p}$-bounded if $p>1+1 / 2 \alpha$.

Proof. From Proposition 2.1, $\left(R_{n, z} \mu\right)^{\#}$ is $L^{p}$-bounded for all $p$ in $(1, \infty]$, as long as $\operatorname{Re}(z) \geq 1$; indeed this operator is dominated by the Hardy-Littlewood maximal operator. On the other hand; if $\operatorname{Re}(z)=\beta>\frac{1}{2}-\alpha$, then Lemma 1.2 implies that, for some $\varepsilon$ in $\mathbf{R}^{+}$,

$$
\left|\left(R_{n, z} \mu\right)^{\uparrow}(\rho \sigma)\right| \leq C_{z}(1+\rho)^{-1 / 2-\varepsilon}, \quad \sigma \in S^{n-1}, \rho \in \mathbf{R}^{+},
$$

and Theorem 1.4 implies that $\left(R_{n, z} \mu\right)^{\#}$ is $L^{2}$-bounded. The constant $C_{z}$ grows exponentially in $z$, so that Stein's complex interpolation method can be applied, to deduce that $\left(R_{n, 0} \mu\right)^{\#}$ is $L^{p}$-bounded for $p$ in $(1+1 / 2 \alpha, 2]$. Since $\mu=R_{n, 0} \mu$, we are done.

3. $L^{p}$-estimates. $2<p<\infty$. Let $S$ be a surface of the sort considered in the preceding section, let $\mu$ be ordinary surface measure on $S$, and let $h: \mathbf{R}^{n} \backslash\{0\} \rightarrow$ $\mathbf{R}^{+} \cup\{0\}$ be bounded, Borel and homogeneous of degree 0 . We suppose that $\mu(\{x \in S: h(x)=0\})=0$. For $z$ in $\mathbf{C}$, denote by $h_{z}$ the function

$$
h_{z}(x)= \begin{cases}{[h(x)]^{z},} & h(x) \neq 0, \\ 0, & h(x)=0 .\end{cases}
$$

Then the family of measures $\mu_{z}$, where $d \mu_{z}=h_{z} d S$, is analytic. More precisely, there exists $c$ in $\mathbf{R}^{+} \cup\{0\}$ such that if $\operatorname{Re}(z)>-c$, then $\left.h_{z}\right|_{S}$ is integrable relative to surface measure: the measures $\mu_{z}$ are analytic for these values of $z$. The number $c$ depends on $h$.

Proposition 3.1. Suppose that there are real numbers $u, v$ in $(-c, \infty)$ and $p, q$ in $[1, \infty]$ such that $\mu_{u}^{\#}$ is $L^{p}$-bounded and $\mu_{v}^{\#}$ is $L^{q}$-bounded. Then, if $0<\theta<1$, $w=\theta u+(1-\theta) v$ and $1 / r=\theta / p+(1-\theta) / q, \mu_{w}^{\#}$ is $L^{r}$-bounded.

PROOF. We use Stein's complex interpolation method once again. If $\operatorname{Re}(z)=u$, then $\left|\mu_{z} * f\right| \leq\left|\mu_{z}\right| *|f|=\mu_{u} *|f|$, so $\mu_{z}^{\#} f \leq \mu_{u}^{\#}|f|$. Therefore $\mu_{z}^{\#}$ is $L^{p}$-bounded 
with norm no greater than that of $\mu_{u}^{\#}$. Similarly, if $\operatorname{Re}(z)=v$, then $\mu_{z}^{\#}$ is $L^{q_{-}}$ bounded with norm at most that of $\mu_{v}^{\#}$. Interpolation implies that $\mu_{w}^{\#}$ is indeed $L^{r}$-bounded.

The main new idea of the first three sections of this paper is incorporated in the following theorem.

THEOREM 3.2. Suppose that for some positive real number a,

$$
\left|\left(\mu_{a}\right) \widehat{\Upsilon}(\rho \sigma)\right| \leq C(1+\rho)^{-1 / 2-\varepsilon}, \quad \sigma \in S^{n-1}, \rho \in \mathbf{R}^{+},
$$

where $\varepsilon>0$. Then $\mu^{\#}$ is $L^{p}$-bounded if $p>2+2 a / c$.

PROOF. If $z>-c$, then $\mu_{z}^{\#}$ is $L^{\infty}$-bounded because $\mu_{z}$ is a bounded measure: on the other hand, by Theorem $1, \mu_{a}^{\#}$ is $L^{2}$-bounded. By Proposition 3.1, it follows that $\mu_{0}^{\#}=\mu^{\#}$ is $L^{p}$-bounded for $p$ in $(2+2 a / c, \infty]$.

4. First examples. In this section, we estimate the Fourier transforms of a particular family of hypersurfaces in $\mathbf{R}^{3}$, with weighting factors. These will illustrate the difficulties involved in the general problem of controlling Fourier transforms of measures supported by hypersurfaces.

Revolve the curve $|x|^{2 a}+|z|^{2 b}=1$ about the $z$-axis in $\mathbf{R}^{3}$. The surface of revolution obtained is given, in cylindrical coordinates $(r, \theta, z)$, by $r^{2 a}+|z|^{2 b}=1$, and the element of surface area is $d S=r d \theta d l$, where $d l^{2}=d r^{2}+d z^{2}$, i.e. $d l$ "is" the line element on the curve. We suppose that $a, b>\frac{1}{2}$; the surface is then convex, differentiable, and smooth except perhaps where $r=0$ or $z=0$. The Gaussian curvature $\kappa$ of the surface is approximately $(2 a-1)(a / b)^{2} r^{4 a-4}$ near $r=0$ and $(2 b-1)(b / a)|z|^{2 b-2}$ near $z=0$.

Let $\mu$ be the surface measure carried by $S$, and let $\mu_{s, t}$ (for complex $s$ and $t$ ) be the measure with $d \mu_{s, t}=r^{s}|z|^{t} d \mu$. With a view to applying the results of the previous sections, we shall study the behaviour of the Fourier transform of $\mu_{s, t}$. The cylindrical symmetry present means that it suffices to look at $\hat{\mu}_{s, t}(\xi, 0, \eta)$. In order to avoid chasing factors of $2 \pi$, we consider the integral

$$
I(\xi, \eta)=\iint_{S} \exp (i[\xi x+\eta z]) r^{s}|z|^{t} d S,
$$

where $s$ and $t$ are complex. It is routine to check that

$$
\iint_{S} \exp (i[\xi z+\eta z]) d S<\infty
$$

as long as $\operatorname{Re}(s)>-2$ and $\operatorname{Re}(t)>-1$. For such values of $s$ and $t, I(\xi, \eta)$ is uniformly bounded as $\xi$ and $\eta$ vary. It is possible to continue $I(\xi, \eta)$ meromorphically in $s$ and $t$, by standard arguments.

For integral $a$ and $b$, the surface is analytic: stationary phase arguments then indicate that, for $s=t=0, I(\rho \sin \omega, \rho \cos \omega)$ should decay as $\rho^{-1}(\rho \rightarrow \infty)$, as long as $\sin \omega \cos \omega \neq 0$, and further suggest that "damping" $d S$ by a factor of $\kappa^{1 / 2}$ might have the effect of producing decay which is uniform in all directions. This turns out to be correct, though not for all nonintegral values of $a$ and $b$. (Further, the corresponding result in higher dimensions is false.) Since we want estimates which hold uniformly in $\omega$, we seek only estimates of decay like $\rho^{-1}$, since these are best possible uniformly, even though in special directions faster decay may occur. 
In this section, we consider $a, b$ to be fixed, and $\xi, \eta$ to be variable. More generally, unless otherwise specified, Greek parameters $(\xi, \eta, \sigma, \tau, \ldots)$ are assumed to be real and to vary over all their range in inequalities. Thus the inequality $\left|I_{0}(\xi, \eta)\right| \leq C(1+|\xi|)^{-1}$ holds uniformly in $\xi$ and $\eta ; C$ may however depend on $a$ and $b$.

Take nonnegative $D(\mathbf{R})$-functions $h_{1}, h_{0}$ and $h_{-1}$ such that $h_{j}(z)=1$ for $z$ in a small neighborhood of $j$ and $h_{j}(z)=0$ if $|z-j| \geq 1(j=0, \pm 1)$, and such that $h_{1}^{2}(z)+h_{0}^{2}(z)+h_{-1}^{2}(z)=1$ for all $z$ in a neighbourhood of $[-1,1]$. Then $I=I_{1}+I_{0}+I_{-1}$, where

$$
I_{j}(\xi, \eta)=\iint_{S} \exp (i[\xi x+\eta z]) r^{s}|z|^{t} h_{j}^{2}(z) d S .
$$

We shall show how to estimate $I_{1}$ and $I_{0}: I_{-1}$ is essentially the same as $I_{1}$. This will have the effect of separating out the contributions of the different singularities.

Proposition 4.1. Suppose that $\operatorname{Re}(s) \geq 2 a-2$. Then

$$
\left|I_{1}(\rho \sin \omega, \rho \cos \omega)\right| \leq C_{s, t}(1+\rho)^{-1} .
$$

The constant $C_{s, t}$ grows at most polynomially in $|s|$ and $|t|$ if $\operatorname{Re}(s)$ and $\operatorname{Re}(t)$ are restricted to compact subintervals of $[2 \alpha-2, \infty)$ and $(-\infty, \infty)$.

Proof. The interval $I_{1}$ is obviously bounded. We note that $z>0$ in the region of effective integration. We show first that

$$
\left|\eta I_{1}(\xi, \eta)\right| \leq C
$$

and then that

$$
\left|\xi I_{1}(\xi, \eta)\right| \leq C
$$

Clearly, we have, for appropriately defined $g$,

$$
\begin{aligned}
d l / d z & =\left(1+(d r / d z)^{2}\right)^{1 / 2}=-(d r / d z)\left(1+(d r / d z)^{-2}\right)^{1 / 2} \\
& =(b / a) r^{1-2 a} z^{2 b-1}(1+g(z)) .
\end{aligned}
$$

Therefore if $s=2 a-2+i \sigma$ (with $\sigma$ in $\mathbf{R}$, by convention)

$$
\begin{aligned}
& \eta I_{1}(\xi, \eta)= \eta \int_{0}^{1} \int_{0}^{2 \pi} \exp (i[\xi r \cos \theta+\eta z]) r^{s+1} z^{t} h_{1}^{2}(z)(d l / d z) d \theta d z \\
&=(2 \pi b \eta / a)\left\{\int_{0}^{1} e^{i \eta z} J_{0}(\xi r) r^{i \sigma} z^{2 b-1+t} h_{1}^{2}(z) d z\right. \\
&\left.\quad+\int_{0}^{1} e^{i \eta z} J_{0}(\xi r) r^{i \sigma} z^{2 b-1+t} h_{1}^{2}(z) g(z) d z\right\} .
\end{aligned}
$$

We regard the first integral as the Fourier transform of the (pointwise) product of the functions

$$
z \rightarrow 1_{(0,1)}(z) J_{0}(\xi r) r^{i \sigma}, \quad z \rightarrow z^{2 b-1+t} h_{1}(z) \text { and } z \rightarrow h_{1}(z),
$$

and the second integral as the Fourier transform of the product of the functions

$$
z \rightarrow 1_{(0,1)}(z) J_{0}(\xi r) r^{i \sigma}, \quad z \rightarrow z^{2 b-1+t} h_{1}(z) \text { and } z \rightarrow h_{1}(z) g(z) .
$$


Here $1_{A}$ is the indicator or characteristic function of the set $A$. Since the Fourier transform of the product is the convolution of the Fourier transforms, it suffices, in order to prove (4.1), to show that, for some positive $\varepsilon$,

$$
\begin{gathered}
\left|\int_{0}^{2} e^{i \eta z} h_{1}(z) d z\right| \leq C(1+|\eta|)^{-2} \\
\left|\int_{0}^{1} e^{i \eta z} h_{1}(z) g(z) d z\right| \leq C(1+|\eta|)^{-1-\varepsilon}, \\
\left|\int_{0}^{2} e^{i \eta z} h_{1}(z) z^{2 b-1+t} d z\right| \leq C(1+|t|)^{2}(1+|\eta|)^{-2}, \\
\left|\int_{0}^{1} e^{i \eta z} J_{0}(\xi r) r^{i \sigma} d z\right| \leq C(1+|\sigma|)(1+|\eta|)^{-1},
\end{gathered}
$$

uniformly for $t$ in $\mathbf{C}$ with bounded real part. The estimates (4.4) and (4.6) are standard. In (4.5), the integral is $C^{\infty}$ except at $z=1$. Now for $z$ close to 1 ,

$$
\begin{aligned}
g(z) & =\left(1+(a / b) r^{2 a-1} z^{1-2 b}\right)^{1 / 2}-1 \\
& =\sum_{k=1}^{\infty} a_{k}\left(r^{2 a-1} z^{1-2 b}\right)^{k} \\
& =\sum_{k=1}^{\infty} a_{k}\left(1-z^{2 b}\right)^{k-k / 2 a}\left(1-\left[1-z^{2 b}\right]\right)^{-k+k / 2 b} \\
& =\sum_{k=1}^{\infty} \sum_{j=0}^{\infty} a_{k} b_{k j}\left(1-z^{2 b}\right)^{k(1-1 / 2 a)+j}
\end{aligned}
$$

The standard techniques of asymptotic analysis now show that

$$
\left|\int_{0}^{1} e^{i \eta z} h_{1}(z) g(z) d z\right| \leq C(1+|\eta|)^{-2+1 / 2 a} .
$$

Inequality (4.7) is rather more delicate: clearly the integral is bounded. Integration by parts shows that

$$
\begin{aligned}
-i \eta \int_{0}^{1} e^{i \eta z} J_{0}(\xi r) r^{i \sigma} d z= & \xi \int_{0}^{1} e^{i \eta z} J_{0}^{\prime}(\xi r) r^{i \sigma}(d r / d z) d z \\
& +\lim _{\delta \rightarrow 0+} i \sigma \int_{0}^{1} e^{i \eta z} J_{0}(\xi r) r^{i \sigma+\delta-1}(d r / d z) d z .
\end{aligned}
$$

We control the first integral by Lemma A.1 (in the Appendix) and the second by Lemma A.2, thereby proving (4.7).

If $\operatorname{Re}(s)=2 a-2+\gamma$, with $\gamma$ in $\mathbf{R}^{+}$, we argue slightly differently. We write

$$
\eta I_{1}(\xi, \eta)=(2 \pi b \eta / a) \int_{0}^{1} e^{i \eta z} J_{0}(\xi r) r^{\gamma+i \sigma} z^{2 b-1+t} h_{1}^{2}(z)[1+g(z)] d z
$$

and regard the integral as the Fourier transform of the product of the functions

$$
z \rightarrow 1_{(0,1)}(z) J_{0}(\xi r) r^{i \sigma}, \quad z \rightarrow z^{2 b-1+t} h_{1}(z)
$$


and

$$
z \rightarrow 1_{(0,1)}(z) r^{\gamma} h_{1}(z)[1+g(z)] .
$$

We use inequalities (4.6), (4.7), and the new inequality

$$
\left|\int_{0}^{1} e^{i \eta z} r^{\gamma} h_{1}(z)[1+g(z)] d z\right| \leq C_{\gamma}(1+|\eta|)^{-1-\varepsilon}
$$

for some positive $\varepsilon$, which is proved in the same way as (4.5), to establish the result in this case.

At this point, we have proved (4.1) with a constant $C$ which behaves reasonably if $\operatorname{Re}(s)=2 a-2$, or if $\operatorname{Re}(s)>2 a-2$, but in the latter case the constant involves the constant $C_{\gamma}$ in inequality (4.8), which blows up as $\operatorname{Re}(s) \rightarrow 2 a-2$. We therefore need to use the Phragmén-Lindelöf principle to conclude that the constant in (4.1) has the growth properties enunciated.

We shall now prove (4.2), by simpler reasoning. From (4.3),

$$
d l / d z=-(d r / d z)[1+g(z)]
$$

so

$$
I_{1}(\xi, \eta)=-2 \pi \int_{0}^{1} e^{i \eta z} J_{0}(\xi r) r^{s+1} z^{t} h_{1}^{2}(z)(d r / d z)[1+g(z)] d z .
$$

The integral is the Fourier transform of the product of the functions

$$
z \rightarrow 1_{(0,1)}(z) J_{0}(\xi r) r^{i \sigma}(d r / d z), \quad z \rightarrow z^{t} h_{1}(z),
$$

and

$$
z \rightarrow 1_{(0,1)}(z) r^{s+1-i \sigma}[1+g(z)] h_{1}(z)
$$

As before, it suffices to show, for some positive $\varepsilon$, that

$$
\begin{aligned}
& \left|\int_{0}^{1} e^{i \eta z} r^{s+1-i \sigma}[1+g(z)] h_{1}(z) d z\right| \leq C(1+|\eta|)^{-1-\varepsilon} \\
& \left|\int_{0}^{2} e^{i \eta z} z^{t} h_{1}(z) d z\right| \leq C(1+|t|)^{2}(1+|\eta|)^{-2} \\
& \left|\int_{0}^{1} e^{i \eta z} J_{0}(\xi r) r^{i \sigma} d r / d z d z\right| \leq C(1+|\xi|)^{-1}
\end{aligned}
$$

The first two inequalities are easy, and (4.10) follows from Lemma A.1.

We now study the integral $I_{1}$ with a more singular weight function.

PROPOSITION 4.2. For fixed $\xi$ and $\eta$, the function

$$
s \rightarrow(s+2) \iint_{S} \exp (i[\xi x+\eta z]) r^{s}|z|^{t} h_{1}^{2}(s) d S
$$

continues analytically into a neighbourhood of $\operatorname{Re}(s) \geq-2$. If $\operatorname{Re}(s) \geq-2$, then

$$
\left|(s+2) I_{1}(\xi, \eta)\right| \leq C_{s, t} .
$$

The constant $C_{s, t}$ grows at most polynomially in $|s|$ and $|t|$ if $\operatorname{Re}(s)$ and $\operatorname{Re}(t)$ are restricted to compact subintervals of $[-2, \infty)$ and $(-\infty, \infty)$.

PROOF. With $k$ in $D(\mathbf{R})$ chosen so that $k(r)=h_{1}^{2}(z)$,

$$
\begin{aligned}
(s+2) & I_{1}(\xi, \eta) \\
= & (s+2) \int_{0}^{2 \pi} \int_{0}^{1} \exp (i[\xi r \cos \theta+\eta z]) r^{s+1} z^{t} k(r)\left(1+(d z / d r)^{2}\right)^{1 / 2} d r d \theta .
\end{aligned}
$$


It will suffice to show that

$$
s \rightarrow(s+2) \int_{0}^{1} \exp (i[\xi r+\eta z]) r^{s+1} z^{t} k(r)\left(1+(d z / d r)^{2}\right)^{1 / 2} d r
$$

continues analytically in $s$ and is bounded (uniformly in $\xi$ and $\eta$ ) when $\operatorname{Re}(s) \geq-2$. By arguing as in the proof of Proposition 4.1, we can reduce matters to showing that

$$
s \rightarrow(s+2) \int_{0}^{1} \exp (i[\xi r+\eta z]) r^{s+1} d r
$$

has these properties. Now

$$
\begin{aligned}
(s+2) & \int_{0}^{1} \exp (i[\xi r+\eta z]) r^{s+1} d r \\
= & (s+2) \int_{0}^{1} e^{i \xi r} e^{i \eta} r^{s+1} d r+(s+2) \int_{0}^{1} e^{i \xi r} e^{i \eta}\left[e^{i \eta(z-1)}-1\right] r^{s+1} d r
\end{aligned}
$$

the first integral is known to continue analytically into $\operatorname{Re}(s)>-3$, while the second continues analytically into $\operatorname{Re}(s)>-2-2 a$, because the integrand is $O\left(\eta r^{s+1+2 a}\right)$, so the analytic continuation is established. When $\operatorname{Re}(s) \geq-1$,

$$
\left|(s+2) \int_{0}^{1} \exp (i[\xi r+\eta z]) r^{s+1} d r\right| \leq|s+2|
$$

if we can establish that

$$
\left|i \sigma \int_{0}^{1} \exp (i[\xi r+\eta z]) r^{i \sigma-1} d r\right| \leq C(1+|\sigma|),
$$

then the Phragmén-Lindelöf principle will imply the required boundedness.

To prove (4.12), we let $\gamma$ be $\min \left\{1,|\eta|^{-1 / 2 a}\right\}$, and write, as in (4.11),

$$
\begin{aligned}
i \sigma \int_{0}^{1} \exp (i[\xi r+\eta z]) r^{i \sigma-1} d r= & i \sigma \int_{0}^{\gamma} e^{i \xi r} e^{i \eta} r^{i \sigma-1} d r \\
& +i \sigma \int_{0}^{\gamma} e^{i \xi r} e^{i \eta}\left[e^{i \eta(z-1)}-1\right] r^{i \sigma-1} d r \\
& +i \sigma \int_{\gamma}^{1} e^{i \xi r} e^{i \eta z} r^{i \sigma-1} d r
\end{aligned}
$$

The first integral is known to be bounded uniformly in $\xi$ and $\eta$ and the second is easily checked to be bounded too. To estimate the third, we write

$$
\begin{aligned}
\left|\int_{\gamma}^{1} e^{i \xi r} e^{i \eta z} r^{i \sigma-1} d r\right| & =\left|\int_{\gamma}^{1} \int_{r}^{\infty} e^{i \xi r} e^{i \eta z}(1-i \sigma) x^{i \sigma-2} d x d r\right| \\
& =\left|(1-i \sigma) \int_{\gamma}^{\infty} x^{i \sigma-2} \int_{\gamma}^{\min (1, x)} e^{i \xi r} e^{i \eta z} d r d x\right| \\
& \leq(1+|\sigma|) \int_{\gamma}^{\infty} x^{-2}\left|\int_{\gamma}^{\min (1, x)} e^{i \xi r} e^{i \eta z} d r\right| d x
\end{aligned}
$$

From Lemma A.3, we know the inner integral is $O(1+|\eta|)^{-1 / 2 a}$, and inequality (4.12) follows. 
COROLlaRY 4.3. If $-1 \leq \gamma \leq 0$, and $\operatorname{Re}(s) \geq-2(a \gamma+1), s \neq-2$, then

$$
\left|I_{1}(\rho \sin \omega, \rho \cos \omega)\right| \leq C_{s, t}(1+\rho)^{\gamma} \text {. }
$$

PROOF. This corollary follows by interpolating between the results of Propositions 4.1 and 4.2 , and using the analytic function

$$
s \rightarrow(s+2) I_{1}(\rho \sin \omega, \rho \cos \omega) \text {. }
$$

We now turn our hands to the study of the integral $I_{0}$, which is treated by similar techniques. The results are less simple to state, but no more difficult to prove. We need the following notation: $c$ and $A$ are chosen so that

$$
\operatorname{supp}\left(h_{0}\right) \subset[-c, c] \subseteq(-1,1), \quad A=3 \max \left\{\left|r^{\prime}(z)\right|: z \in[-c, c]\right\} .
$$

Proposition 4.4. (i) Suppose that $\operatorname{Re}(t) \geq b-1$. Then

$$
\left|I_{0}(\xi, \eta)\right| \leq C_{s, t}(1+|\xi|)^{-1} .
$$

(ii) Suppose that $\operatorname{Re}(t) \geq 0$. Then $\left|I_{0}(\xi, \eta)\right| \leq C_{s, t}(1+|\eta|)^{-1}$ if $|\xi| \leq A|\eta|$.

The constants $C_{s, t}$ grow at most polynomially in $|s|$ and $|t|$ if $\operatorname{Re}(s)$ and $\operatorname{Re}(t)$ are restricted to compact subintervals of $(-\infty, \infty)$ and $[b-1, \infty)$ in (i) and $(-\infty, \infty)$ and $[0, \infty)$ in (ii).

ProOF OF (i). We write

$$
I_{0}(\xi, \eta)=2 \pi \int_{-1}^{+1} e^{i \eta z} J_{0}(\xi r) r^{s+1}|z|^{t} h_{0}^{2}(z)(d l / d z) d z
$$

and consider the integral to be the Fourier transform of the product of the functions

$$
z \rightarrow 1_{(-1,1)}(z) J_{0}(\xi r) r^{1 / 2}|z|^{b-1+i \tau}
$$

and

$$
z \rightarrow 1_{(-1,1)}(z) r^{s+1 / 2}|z|^{\gamma}(d l / d z) h_{0}^{2}(z),
$$

where $\gamma=t+1-i \tau-b$. Using the same techniques as in the proofs of Propositions 4.1 and 4.2 , we can show that, for some $\varepsilon$ in $\mathbf{R}^{+}$,

$$
\left.\left|\int_{-1}^{+1} e^{i \eta z} r^{s+1 / 2}\right| z\right|^{\gamma}(d l / d z) h_{0}^{2}(z) d z \mid \leq C_{s, t}(1+|\eta|)^{-1-\varepsilon},
$$

and Lemma A.4 implies that

$$
\left.\left|\int_{-1}^{+1} e^{i \eta z} J_{0}(\xi r) r^{1 / 2}\right| z\right|^{b-1+i \tau} d z \mid \leq C_{\tau}(1+|\eta|)^{-1}
$$

the first part of the proposition follows.

PROOF OF (ii). In this case, we write

$$
I_{0}(\xi, \eta)=\int_{0}^{2 \pi} \int_{-1}^{+1} \exp (i[\xi r \cos \theta+\eta z]) r^{s+1}|z|^{t} h_{0}^{2}(z)(d l / d z) d z d \theta
$$

The inner integral is the Fourier transform of the products of the functions

$$
z \rightarrow 1_{(-c, c)}(z) \exp (i \xi r \cos \theta)|z|^{i \tau}
$$

and

$$
z \rightarrow r^{s+1}|z|^{t-i \tau}(d l / d z) h_{0}^{2}(z)
$$


Lemma A.5 implies that

$$
\left.\left|\int_{-c}^{c} \exp (i[\xi r \cos \theta+\eta z])\right| z\right|^{i \tau} d z \mid \leq C_{\tau}(1+|\eta|)^{-1},
$$

and it is easy to check that

$$
\left.\left|\int_{-1}^{+1} e^{i \eta z} r^{s+1}\right| z\right|^{t-\tau}(d l / d z) h_{0}^{2}(z) \mid \leq C_{s, t}(1+|\eta|)^{-1-\varepsilon}
$$

for some positive $\varepsilon$; the second part of the proposition follows.

We continue our examination of Fourier transforms with the following proposition.

Proposition 4.5. For fixed $\xi$ and $\eta$, the function

$$
t \rightarrow(t+1) \iint_{S} \exp (i[\xi x+\eta z]) r^{s}|z|^{t} h_{0}^{2}(z) d S
$$

continues analytically into a neighbourhood of $\operatorname{Re}(t) \geq-1$. If $\operatorname{Re}(t) \geq-1$, then

$$
\left|(t+1) I_{0}(\xi, \eta)\right| \leq C_{s, t}(1+|\xi|)^{-1 / 2}
$$

the constant $C_{s, t}$ grows at most polynomially in $|s|$ and $|t|$ if $\operatorname{Re}(s)$ and $\operatorname{Re}(t)$ are restricted to compact subintervals of $(-\infty, \infty)$ and $[-1, \infty)$.

ProOF. To establish the analytic continuation, it suffices to find the continuation of

$$
\begin{aligned}
(t+1) & \int_{-c}^{+c} \exp (-i[\xi r \cos \theta+\eta z])|z|^{t} d z \\
= & (t+1) \int_{-c}^{+c} e^{i \eta z} e^{i \xi \cos \theta}|z|^{t} d z \\
& +(t+1) \int_{-c}^{+c} e^{i \eta z} e^{i \xi \cos \theta}\left[e^{i \xi \cos \theta(r-1)}-1\right]|z|^{t} d t
\end{aligned}
$$

and both these expressions continue analytically. (Compare with the proof of Proposition 4.2.) In order to obtain the estimate enunciated, it suffices to show that

$$
\left.\left|i \tau \int_{-c}^{c} e^{i \eta z} J_{0}(\xi r) r^{1 / 2}\right| z\right|^{i \tau-1} d z \mid \leq C_{\tau}(1+|\xi|)^{-1 / 2}
$$

We write $\gamma=\min \left\{c,|\xi|^{-1 / 2 b}\right\}$. Then

$$
\begin{aligned}
i \tau \int_{-c}^{c} & e^{i \eta z} J_{0}(\xi r) r^{1 / 2}|z|^{i \tau-1} d z \\
\quad & i \tau \int_{-\gamma}^{\gamma} e^{i \eta z} J_{0}(\xi) r^{1 / 2}|z|^{i \tau-1} d z \\
& +i \tau \int_{-\gamma}^{\gamma} e^{i \eta z}\left[J_{0}(\xi r)-J_{0}(\xi)\right] r^{1 / 2}|z|^{i \tau-1} d z \\
& +i \tau\left(\int_{-c}^{-\gamma}+\int_{\gamma}^{c}\right) e^{i \eta z} J_{0}(\xi r) r^{1 / 2}|z|^{i \tau-1} d z
\end{aligned}
$$


The first of these terms is known to satisfy

$$
\left.\left|i \tau \int_{-\gamma}^{\gamma} e^{i \eta z} J_{0}(\xi) r^{1 / 2}\right| z\right|^{i \tau-1} d z\left|\leq C_{r}\right| J_{0}(\xi) \mid \leq C_{\tau}(1+|\xi|)^{-1 / 2}
$$

The second is easily estimated: since, for some $s$ in $[r, 1]$

$$
\begin{gathered}
\left|J_{0}(\xi r)-J_{0}(\xi)\right|=\left|J_{0}^{\prime}(\xi s) \xi(r-1)\right| \leq C|\xi|^{-1 / 2}|\xi||z|^{2 b} \\
\left.\left|i \tau \int_{-\gamma}^{\gamma} e^{i \eta z}\left[J_{0}(\xi r)-J_{0}(\xi)\right] r^{1 / 2}\right| z\right|^{i \tau-1} d z \mid \\
\leq C_{\tau} \int_{-\gamma}^{\gamma}|\xi|^{1 / 2}|z|^{2 b-1} d z \leq C_{\tau}(1+|\xi|)^{-1 / 2}
\end{gathered}
$$

Therefore, in order to prove (4.13), it will, by symmetry, be enough to show that, for any $\gamma$ in $[0,1]$,

$$
\left|i \tau \int_{\gamma}^{1} e^{i \eta z} J_{0}(\xi r) r^{1 / 2} z^{i \tau-1} d z\right| \leq C_{\tau}(1+|\xi|)^{-1 / 2}
$$

Now, for $\xi$ big

$$
J_{0}(\xi r)=C_{+}|\xi r|^{-1 / 2} e^{i \xi r}+C_{-}|\xi r|^{-1 / 2} e^{-i \xi r}+O\left(|\xi r|^{-1}\right)
$$

and

$$
\begin{aligned}
& \left|i \tau \int_{\gamma}^{1} e^{i \eta z} O\left(|\xi r|^{-1}\right) r^{1 / 2} z^{i \tau-1} d z\right| \\
& \quad \leq C|\tau| \int_{\gamma}^{1}|\xi r|^{-1} r^{1 / 2} z^{-1} d z \leq C|\xi|^{-1}|\tau|(1+|\log (\gamma)|) \leq C|\tau|(1+|\xi|)^{-1 / 2} .
\end{aligned}
$$

By symmetry, it suffices to establish the estimate

$$
\left|\int_{\gamma}^{1} e^{i \eta z} e^{i \xi r} z^{i \tau-1} d z\right| \leq C_{\tau}
$$

to prove (4.14) and thereby the proposition. Now

$$
\begin{aligned}
\left|\int_{\gamma}^{1} e^{i \eta z} e^{i \xi r} z^{i \tau-1} d z\right| & =\left|\int_{\gamma}^{1} \int_{z}^{\infty} e^{i \eta z} e^{i \xi r}(1-i \tau) x^{i \tau-2} d x d z\right| \\
& =\left|(1-i \tau) \int_{\gamma}^{\infty} \int_{\gamma}^{\min \{x, 1\}} e^{i \eta z} e^{i \xi r} x^{i \tau-2} d z d x\right| \\
& \leq(1+|\tau|) \int_{\gamma}^{\infty} x^{-2}\left|\int_{\gamma}^{\min \{x, 1\}} e^{i \eta z} e^{i \xi r} d z\right| d x \\
& \leq C(1+|\tau|) \int_{\gamma}^{\infty} x^{-2}|\xi|^{-1 / 2 b} d x=C_{\tau},
\end{aligned}
$$

by Lemma A.3, with the roles of $r$ and $z$ reversed.

COROLLARY 4.6. (i) If $-1 \leq \gamma \leq \frac{1}{2}$ and $\operatorname{Re}(t) \geq-b(2 \gamma+1)-1, t \neq-1$, then $\left|I_{0}(\xi, \eta)\right| \leq C_{s, t}(1+|\xi|)^{\gamma}$.

(ii) If $-1 \leq \gamma \leq 0$ and $\operatorname{Re}(t) \geq-(\gamma+1), t \neq-1$, then $\left|I_{0}(\xi, \eta)\right| \leq C_{s, t}(1+|\eta|)^{\gamma}$ if $|\xi| \leq A|\eta|$.

ProOF. This corollary follows by interpolation between the results of Propositions 4.4 and 4.5 using the analytic function $t \rightarrow(t+1) I_{0}(\xi, \eta)$. 
COROLLARY 4.7. The maximal operator $\mu^{\#}$ is $L^{p}$-bounded when

(i) $1<p \leq 2, p>1+a / 2, p>(2 b+1) /(b+1)$, or

(ii) $2 \leq p<\infty, p>2 a$.

ProOF. We have that $|\hat{\mu}(\rho \sigma)| \leq C(1+\rho)^{\gamma}, \sigma \in S^{2}, \rho \in \mathbf{R}^{+}$, if $\gamma \geq-1 / a$ and $\gamma \geq-1 / 2 b-\frac{1}{2}$ (by Corollaries 4.3 and 4.6); (i) follows from Theorem 2 . To prove (ii), we apply Theorem 3 to the measures $\mu_{s, 0}$ in similar fashion.

Easy examples show that these results are essentially best possible. It may be worth pointing out that the formulation of part (ii) of Theorem III.3 of [1] is wrong; Corollary 4.7 presents the correct result.

5. Further results. Similar examples in higher dimensions can be treated by this method: a new effect can then be observed. Before we describe this, we observe that, in the above example, when the index $b$ lies in $\left(\frac{1}{2}, 1\right)$, then the Fourier transform $\left(\kappa^{1 / 2} \mu\right)^{\uparrow}(0,0, \eta)$ is given by

$$
\begin{aligned}
\left(\kappa^{1 / 2} \mu\right)^{\wedge}(0,0, \eta) & =\iint_{S} e^{i \eta z} \kappa^{1 / 2} d S=\int_{-1}^{+1} e^{i \eta z} \kappa^{1 / 2} 2 \pi r(d l / d z) d z \\
& =\int_{-1}^{+1} e^{i \eta z}|z|^{b-1} h(z) d z
\end{aligned}
$$

where $h$ is reasonably well behaved near 0 . The "singularity at $z=0$ " therefore "propagates in the $z$ direction", in as much as it causes a decay of the order of $|\eta|^{-b}$ in the Fourier transform, and this decay is less rapid than the $|\eta|^{-1}$ decay one might hope for. This phenomenon depends on the unboundedness of $\kappa$, so one might imagine that for nicer surfaces (e.g. analytic surfaces) it might not occur. However, such fancies are idle. We consider the analytic surface $S$ in $\mathbf{R}^{6}$ given by

$$
u^{2}+v^{2}+w^{2}+x^{2}+y^{2}+z^{4}=1 \text {. }
$$

The singularity in the curvature is at $z=0$, where we have (approximately) $\kappa \simeq 3 z^{2}$. In this case,

$$
\left(\kappa^{1 / 2} \mu\right)^{\curlyvee}(0,0,0,0,0, \eta) \simeq \int_{-1}^{+1} e^{i \eta z} \sqrt{3}|z| h(z) d z
$$

where $h$ is reasonably well behaved, and so $\left(\kappa^{1 / 2} \mu\right)^{-}$decays as $|\eta|^{-2}$ because of the singularity of $|z|$ at 0 . This is not as rapid as one would like; indeed, in other directions, the decay is of the order of $\rho^{-5 / 2}$, which is what one would hope for. Even for analytic surfaces, then, there can be "nonnormal propagation of singularities"

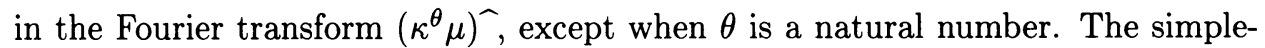
minded conjecture that $\left(\kappa^{1 / 2} \mu\right)$ decays as $\rho^{-(n-1) / 2}, n$ being the dimension of the ambient space, fails.

We conjecture that, for reasonably smooth hypersurfaces, $(\kappa \mu)^{-}$decays as $\rho^{-(n-1) / 2}, n$ being the dimension of the ambient space. At present, the best we can do in general is to show that $\left(\kappa^{\theta} \mu\right)^{\wedge}$ has this decay, where $\theta$ is the integer part of $(n+3) / 2$, though for $n=2$ the methods of the Appendix are sufficiently powerful to show that $\left(\kappa^{1 / 2} \mu\right)^{\wedge}$ decays as $\rho^{-1 / 2}$ if $\mu$ is the arc measure on a compact analytic curve. We also conjecture that if $n \geq 3$ and if $\hat{\mu}$ decays as $\rho^{-\alpha}$, where $\alpha<1$, then 
$\left(\kappa^{\theta} \mu\right)^{\wedge}$ decays as $\rho^{-1}$ when $\theta=(1-\alpha) /(n-1-2 \alpha)$ (the result one would obtain by interpolation if $\left(\kappa^{1 / 2} \mu\right)$ decayed as $\rho^{-(n-1) / 2}$; however, we have only been able to verify this for a few examples, including the example of measures supported on the graph of a homogeneous function whose curvature vanishes at the origin only). Since none of our results is definite, and the proofs are long, we have not included them here, but hope to publish more on these questions soon.

Appendix - van der Corput type lemmata. In this Appendix, we have collected various results on the decay of oscillatory integrals, which use van der Corput's techniques (see, e.g., [7] for an account of these).

We assume that $a, b$ are fixed real numbers, with $a>\frac{1}{2}$ and $b>\frac{1}{2}$; we suppose $0 \leq r \leq 1,0 \leq z \leq 1$ and $r^{2 a}+z^{2 b}=1$, so that $r$ is implicitly a function of $z$ and vice versa. The parameters $\alpha, \beta, \xi, \eta, \sigma$ are arbitrary real numbers, unless further restrictions are specifically indicated. All inequalities hold uniformly as all the (Greek) parameters vary over their ranges.

LEMMA A.1. If $\alpha, \beta \in[0,1]$, then

$$
\left|\xi \int_{\alpha}^{\beta} e^{i \eta z} K(\xi r) r^{i \sigma} d r\right| \leq C(1+|\sigma|)^{1 / 2},
$$

where $K$ denotes either the Bessel function $J_{0}$ or its derivative $J_{0}^{\prime}$.

Proof. We may assume $\alpha=0$ and $\xi>0$, and that $\xi \beta>1$ for

$$
\left|\xi \int_{0}^{\beta} e^{i \eta z} K(\xi r) r^{i \sigma} d r\right| \leq \xi \beta .
$$

Now

$$
K(x)=C_{+}|x|^{-1 / 2} e^{i x}+C_{-}|x|^{-1 / 2} e^{-i x}+O\left(|x|^{-3 / 2}\right)
$$

so

$$
\begin{aligned}
\xi \int_{0}^{\beta} e^{i \eta z} K(\xi r) r^{i \sigma} d r \\
=\xi \int_{0}^{1 / \xi} e^{i \eta z} K(\xi r) r^{i \sigma} d r+\xi \int_{1 / \xi}^{\beta} e^{i \eta z} O\left(|\xi r|^{-3 / 2}\right) r^{i \sigma} d r \\
\quad+C \xi^{1 / 2} \int_{1 / \xi}^{\beta} e^{i \eta z} e^{i \xi r} r^{i \sigma-1 / 2} d r \\
\quad+C^{\prime} \xi^{1 / 2} \int_{1 / \xi}^{\beta} e^{i \eta z} e^{-i \xi r} r^{i \sigma-1 / 2} d r
\end{aligned}
$$

The first two integrals are obviously bounded, and changing $\eta$ and $\sigma$ to $-\eta$ and $-\sigma$ changes the last integral to the complex conjugate of the third. It will therefore suffice to show that (for all $\beta$ in $[0,1]$ )

$$
\left|\xi^{1 / 2} \int_{0}^{\beta} e^{i \eta z} e^{i \xi r} r^{i \sigma-1 / 2} d r\right| \leq C(1+|\sigma|)^{1 / 2}
$$


Put $w=(\xi r)^{1 / 2}, \gamma=(\xi \beta)^{1 / 2}, \psi(w)=\eta z\left(w^{2} / \xi\right)$ and $\phi(w)=\psi(w)+w^{2}+$ $2 \sigma \log w$. Then $\gamma \in\left[0, \xi^{1 / 2}\right]$ and we need to show that

$$
\left|\int_{0}^{\gamma} \exp (i \phi(w)) d w\right| \leq C(1+|\sigma|)^{1 / 2}
$$

which we do using van der Corput's method.

Notice that $\left(w^{3} \phi^{\prime \prime \prime}(w)\right)^{\prime}=\left(w^{3} \psi^{\prime \prime \prime}(w)\right)^{\prime}$, which is of constant sign for $w$ in $[0, \gamma]$. (This can be seen by writing $z\left(w^{2} / \xi\right)$ as a power series in $w$ with all nonconstant terms with negative coefficients, and manipulating this series.) Consequently, $w \rightarrow$ $w^{3} \phi^{\prime \prime \prime}(w)$ and hence $\phi^{\prime \prime \prime}$ vanish at most once in $(0, \gamma), \phi^{\prime \prime}$ vanishes at most twice, and $(0, \gamma)$ can be divided into at most three intervals where $\phi^{\prime}$ is monotone.

Take $c=2(2 a-1) /(12 a-1)$, and let $J$ be the set of $w$ such that $w \geq(|s| / c)^{1 / 2}+1$ and $\left|\phi^{\prime}(w)\right|<c w$. For $w$ in $J, \psi^{\prime}(w)+2 w+2 \sigma / w<c w$ so

$$
\psi^{\prime}(w)<-2 w+c w-2 \sigma / w<-2 w+c w+2 c w .
$$

Since

$$
\begin{gathered}
\psi^{\prime}(w)=-(2 a \eta / b) \xi^{-2 a} w^{4 a-1}\left(1-w^{4 a} / \xi^{2 a}\right)^{1 / 2 b-1} \\
w \psi^{\prime \prime}(w)=\left[(4 a-1)+2 a(2-1 / b) w^{4 a} \xi^{-2 a}\left(1-w^{4 a} / \xi^{2 a}\right)^{-1}\right] \psi^{\prime}(w),
\end{gathered}
$$

so for $w$ in $J$,

$$
\psi^{\prime \prime}(w) \leq(4 a-1) \psi^{\prime}(w) / w<(4 a-1)(3 c-2)
$$

and

$$
\begin{aligned}
\phi^{\prime \prime}(w) & =\psi^{\prime \prime}(w)+2-2 \sigma / w^{2} \\
& <(4 a-1)(3 c-2)+2+2 c=-2(2 a-1)
\end{aligned}
$$

To prove (A.1), integrate over the region where $w<(|\sigma| / c)^{1 / 2}+1$, which contributes at most $(|\sigma| / c)^{1 / 2}+1$ to the total, and break up the rest into integrals over the intervals in which $\phi^{\prime}$ is monotonic. These intervals in turn break up into at most two intervals in which $\phi^{\prime}$ is monotonic and $\left|\phi^{\prime}(w)\right| \geq c w$ (hence also $\left|\phi^{\prime}\right| \geq c$ ) and at most one interval where $\left|\phi^{\prime}(w)\right|<c w$ and so $\left|\phi^{\prime \prime}\right|>2(2 a-1)$. By van der Corput's lemma, (4.1) holds and the lemma is proved.

LemmA A.2. If $\alpha, \beta \in[0,1]$, and $\sigma \neq 0$, then

$$
\lim _{\delta \rightarrow 0+}\left|\int_{\alpha}^{\beta} e^{i \eta z} r^{\delta+i \sigma-1} J_{0}(\xi r) d r\right| \leq C\left(|\sigma|+|\sigma|^{-1}\right) .
$$

PROOF. We may assume $\alpha=0$ and $\xi \geq 0$.

Now $J_{0}(\xi r)=1+O(\xi r)$, so if $\xi \beta \leq 1$, then

$$
\lim _{\delta \rightarrow 0+} \int_{0}^{\beta} e^{i \eta z} r^{\delta+i \sigma-1} J_{0}(\xi r) d r=\lim _{\delta \rightarrow 0+} \int_{0}^{\beta} e^{i \eta z} r^{\delta+i \sigma-1}+O(1)
$$


while if $\xi \beta>1$, then, using also the fact that $J_{0}(\xi r)=O\left(|\xi r|^{-1 / 2}\right)$, we have that

$$
\begin{aligned}
\lim _{\delta \rightarrow 0+} & \int_{0}^{\beta} e^{i \eta z} r^{\delta+i \sigma-1} J_{0}(\xi r) d r \\
= & \lim _{\delta \rightarrow 0+} \int_{0}^{1 / \xi} e^{i \eta z} r^{\delta+i \sigma-1} d r+\lim _{\delta \rightarrow 0+} \int_{0}^{1 / \xi} e^{i \eta z} r^{\delta+i \sigma-1} O(\xi r) d r \\
& +\lim _{\delta \rightarrow 0+} \int_{1 / \xi}^{\beta} e^{i \eta z} r^{\delta+i \sigma-1} O\left(|\xi r|^{-1 / 2}\right) d r \\
= & \lim _{\delta \rightarrow 0+} \int_{0}^{1 / \xi} e^{i \eta z} r^{\delta+i \sigma-1} d r+O(1) .
\end{aligned}
$$

Therefore we need only show that, if $\gamma \in[0,1]$, then

$$
\left|\lim _{\delta \rightarrow 0+} \int_{0}^{\gamma} e^{i \eta z} r^{\delta+i \sigma-1} d r\right| \leq C\left(|\sigma|+|\sigma|^{-1}\right) .
$$

We set $v=r^{2 a}$; then it suffices to show that

$$
\left|\lim _{\delta \rightarrow 0+} \int_{0}^{\gamma} \exp \left(i \eta(1-v)^{1 / 2 b}\right) v^{2 i a \sigma+\delta-1} d v\right| \leq C\left(|\sigma|+|\sigma|^{-1}\right) .
$$

Suppose that $\varepsilon=\min \{\gamma, 2 b /|\eta|\}$. Since

$$
\begin{aligned}
\left|\exp \left(i \eta(1-v)^{1 / 2 b}\right)-\exp (i \eta)\right| & =\left|\exp \left(i \eta\left[(1-v)^{1 / 2 b}-1\right]\right)-1\right| \\
& \leq|\eta|\left|(1-v)^{1 / 2 b}-1\right| \leq|\eta| v
\end{aligned}
$$

we have

$$
\begin{aligned}
& \lim _{\delta \rightarrow 0+}\left|\int_{0}^{\varepsilon} \exp \left(i \eta(1-v)^{1 / 2 b}\right) v^{2 i a \sigma+\delta-1} d v\right| \\
& \quad \leq \lim _{\delta \rightarrow 0+}\left|\int_{0}^{\varepsilon} \exp (i \eta) v^{2 i a \sigma+\delta-1} d v\right|+\lim _{\delta \rightarrow 0+} \int_{0}^{\varepsilon}|\eta| v^{\delta} d v \\
& \quad=1 / 2 a|\sigma|+\varepsilon|\eta| \leq C\left(1+|\sigma|^{-1}\right) .
\end{aligned}
$$

Moreover,

$$
\begin{aligned}
\lim _{\delta \rightarrow 0+} & \int_{\varepsilon}^{\gamma} \exp \left(i \eta(1-v)^{1 / 2 b}\right) v^{2 i a \sigma+\delta-1} d v \\
& =\int_{\varepsilon}^{\gamma} \exp \left(i \eta(1-v)^{1 / 2 b}\right)(1-2 i a \sigma) \int_{v}^{\infty} x^{2 i a \sigma-2} d x d v \\
& =(1-2 i a \sigma) \int_{\varepsilon}^{\infty} x^{2 i a \sigma-2} \int_{\varepsilon}^{\min (x, \gamma)} \exp \left(i \eta(1-v)^{1 / 2 b}\right) d v d x
\end{aligned}
$$

Finally, we have the trivial estimate

$$
\left|\int_{\varepsilon}^{\min (x, \gamma)} \exp \left(i \eta(1-v)^{1 / 2 b}\right) d v\right| \leq \min (x, \gamma)-\varepsilon
$$

and the van der Corput type estimate

$$
\left|\int_{\varepsilon}^{\min (x, \gamma)} \exp \left(i \eta(1-v)^{1 / 2 b}\right) d v\right| \leq 2 b /|\eta|
$$


(this is because $\left|d(1-v)^{1 / 2 b} / d v\right|=\left|(-1 / 2 b)(1-v)^{1 / 2 b-1}\right| \geq 1 / 2 b$ for $v$ in $\left.(0,1)\right)$, so that

$$
\text { (A.4) } \begin{aligned}
\lim _{\delta \rightarrow 0+} & \left|\int_{\varepsilon}^{\gamma} \exp \left(i \eta(1-v)^{1 / 2 b}\right) v^{2 i a \sigma+\delta-1} d v\right| \\
& \leq(1+2 a|\sigma|) \int_{\varepsilon}^{\infty} x^{-2} \min \{\min (x, \gamma)-\varepsilon, 2 b /|\eta|\} d x \leq C(1+|\sigma|) .
\end{aligned}
$$

By combining (A.3) and (A.4) we have proved (A.2) and hence the lemma.

LEMMA A.3. For $\alpha, \beta$ in $[0,1],\left|\int_{\alpha}^{\beta} e^{i \xi r} e^{i \eta z} d r\right| \leq C|\eta|^{-1 / 2 a}$.

PROOF. Without loss of generality, we may assume $\alpha=0$; our integral is then $\int_{0}^{\beta} \exp (i \phi(r)) d r$, where $\phi(r)=\xi r+\eta z(r)$. Now, by writing $z$ as a power series in $r^{2 a}$, we see that

$$
\left|\phi^{\prime \prime}(r)\right|=|\eta|\left|z^{\prime \prime}(r)\right| \geq(2 a-1)(a / b)|\eta| r^{2 a-2} .
$$

Take $\delta=\min \left(\beta,|\eta|^{-1 / 2 a}\right)$. Then

$$
\left|\int_{0}^{\beta} \exp (i \phi(r)) d r\right| \leq\left|\int_{0}^{\delta} \exp (i \phi(r)) d r\right|+\left|\int_{\delta}^{\beta} \exp (i \phi(r)) d r\right| .
$$

The first integral is no bigger than $\delta$ in absolute value, and the second is either 0 (if $\delta=\beta$ ) or can be estimated by van der Corput's lemma, using $\left|\phi^{\prime \prime}(r)\right| \geq$ $C|\eta| \delta^{2 a-2}$.

LEMmA A.4. If $\alpha, \beta \in[0,1]$, then

$$
\left|\xi \int_{\alpha}^{\beta} e^{i \eta z} J_{0}(\xi r) r^{1 / 2} z^{b-1+i \tau} d z\right| \leq C(1+|\tau|) .
$$

PROOF. We may assume that $\alpha=0$ and $\xi \geq 0$. Since

$$
J_{0}(x)=C_{+}|x|^{-1 / 2} e^{i x}+C_{-}|x|^{-1 / 2} e^{-i x}+O\left(|x|^{-1}\right),
$$

we have

$$
\begin{aligned}
\xi \int_{0}^{\beta} e^{i \eta z} J_{0}(\xi r) r^{1 / 2} z^{b-1+i \tau} d z \\
=C_{+} \xi^{1 / 2} \int_{0}^{\beta} e^{i \eta z} e^{i \xi r} z^{b-1+i \tau} d z \\
\quad+C_{-} \xi^{1 / 2} \int_{0}^{\beta} e^{-i \eta z} e^{-i \xi r} z^{b-1+i \tau} d z \\
\quad+\xi \int_{0}^{\beta} e^{i \eta z} O\left(|\xi r|^{-1}\right) r^{1 / 2} z^{b-1+i \tau} d z
\end{aligned}
$$

The last integral is clearly bounded, uniformly in the parameters. It suffices to estimate

$$
\xi^{1 / 2} \int_{0}^{\beta} e^{-i \eta z} e^{-i \xi r} z^{b-1+i \tau} d z
$$


We set $v=z^{b}, \psi(v)=\left(1-v^{2}\right)^{1 / 2 a}$ and $\phi(v)=\eta v^{1 / b}+\xi \psi(v)+b \tau \log (v)$. Then we must prove that, for arbitrary $\beta$ in $[0,1]$,

$$
\left|\xi^{1 / 2} \int_{0}^{\beta} \exp (i \phi(v)) d v\right| \leq C(1+|\tau|)
$$

Now it follows from the definitions of $\phi$ and $\psi$ that

$$
v \phi^{\prime \prime}(v)-(1 / b-1) \phi^{\prime}(v)=\xi\left[v \psi^{\prime \prime}(v)-(1 / b-1) \psi^{\prime}(v)\right]-\tau / v
$$

By writing $\psi(v)$ as a power series in $v^{2}$ with all terms after the constant term with negative coefficients, we see that, if $v \in(0,1)$, then

$$
v \phi^{\prime \prime}(v)-(1 / b-1) \psi^{\prime}(v) \leq(1-1 / a)(2-1 / b) v,
$$

so

$$
\left|v \phi^{\prime \prime}(v)-(1 / b-1) \phi^{\prime}(v)\right| \leq(\xi / a)(2-1 / b) v-|\tau| / v .
$$

Let $\gamma=[2 a b(1+|\tau|) /(2 b-1) \xi]^{1 / 2}$. If $\gamma \geq \beta$, then

$$
\left|\xi^{1 / 2} \int_{0}^{\beta} \exp (i \phi(v)) d v\right| \leq \xi^{1 / 2} \beta \leq C(1+|\tau|)^{1 / 2}
$$

if $\gamma<\beta$, then we divide the integral in (A.5) in two:

$$
\begin{aligned}
& \left|\xi^{1 / 2} \int_{0}^{\beta} \exp (i \phi(v)) d v\right| \\
& \quad \leq\left|\xi^{1 / 2} \int_{0}^{\gamma} \exp (i \phi(v)) d v\right|+\left|\xi^{1 / 2} \int_{\gamma}^{\beta} \exp (i \phi(v)) d v\right| \\
& \quad \leq C(1+|\tau|)^{1 / 2}+\left|\xi^{1 / 2} \int_{\gamma}^{\beta} \exp (i \phi(v)) d v\right|
\end{aligned}
$$

From (A.6), if $v \in[\gamma, \beta]$,

$$
\left|v \phi^{\prime \prime}(v)-(1 / b-1) \phi^{\prime}(v)\right| \geq(\xi / 2 a)(2-1 / b) v .
$$

If $b=1$, then we conclude that $\left|\phi^{\prime \prime}(v)\right| \geq C$, so that

$$
\left|\xi^{1 / 2} \int_{\gamma}^{\beta} \exp (i \phi(v)) d v\right| \leq C
$$

by van der Corput's lemma. If $b \neq 1$, we must argue more deviously: clearly

$$
\left|v \phi^{\prime \prime}(v)\right| \geq(\xi / 4 a)(2-1 / b) v, \quad \text { or } \quad\left|(1 / b-1) \phi^{\prime}(v)\right| \geq(\xi / 4 a)(2-1 / b) v ;
$$

alternatively,

$$
\left|\phi^{\prime \prime}(v)\right| \geq(\xi / 4 a)(2-1 / b), \quad \text { or } \quad\left|\phi^{\prime}(v)\right| \geq(\xi / 4 a)(2-1 / b)|1 / b-1|^{-1} \gamma .
$$

Because

$$
\left(v^{1-1 / b}\left(v^{2} \phi^{\prime \prime}(v)\right)^{\prime}\right)^{\prime}=\xi\left(v^{1-1 / b}\left(v^{2} \psi^{\prime \prime}(v)\right)^{\prime}\right)^{\prime}
$$

and this latter expression is of constant sign, $v^{1-1 / b}\left(v^{2} \phi^{\prime \prime}(v)\right)^{\prime}$ and so also $\left(v^{2} \phi^{\prime \prime}(v)\right)^{\prime}$ have at most one zero in $(0,1)$, and then $v^{2} \phi^{\prime \prime}(v)$ and so also $\phi^{\prime \prime}(v)$ have at most 
two zeros in $(0,1)$. Thus there are at most three subintervals of $[\gamma, \beta]$ in which $\phi^{\prime}$ is monotone; each of these subintervals breaks up into at most two subintervals in which $\phi^{\prime}$ is monotone and

$$
\begin{aligned}
\left|\phi^{\prime}(v)\right| & \geq(\xi / 4 a)(2-1 / b)|1 / b-1|^{-1} \gamma \\
& \geq C \xi^{1 / 2}(1+|\tau|)^{1 / 2}
\end{aligned}
$$

and a third subinterval in which

$$
\left|\phi^{\prime \prime}(v)\right| \geq(\xi / 4 a)(2-1 / b)=C \xi .
$$

Application of van der Corput's lemma now shows that

$$
\left|\xi^{1 / 2} \int_{\gamma}^{\beta} \exp (i \phi(v)) d v\right| \leq C \xi^{1 / 2},
$$

and (A.5) is proved, and thereby the lemma.

LEMMA A.5. Suppose that $\alpha \in(0,1)$ and that $A=3 \max \left\{\left|r^{\prime}(z)\right|: z \in[0, \alpha]\right\}$. Then

$$
\left|\eta \int_{0}^{\alpha} \exp (i[\xi r+\eta z]) z^{i \tau} d z\right| \leq C(1+|\tau|)
$$

for $\xi$ and $\eta$ such that $|\eta| \geq A|\xi|$.

PROOF. The integral is $\int_{0}^{\alpha} \exp (i \phi(z)) d z$, where $\phi(z)=\xi r+\eta z+\tau \log (z)$. Take $\gamma=3|\tau / \eta|$. If $\gamma \geq \alpha$, then

$$
\left|\eta \int_{0}^{\alpha} \exp (i \phi(z)) d z\right| \leq|\eta| \alpha \leq 3|\tau|
$$

while if $\gamma<\alpha$, then

$$
\left|\eta \int_{0}^{\alpha} \exp (i \phi(z)) d z\right| \leq 3|\tau|+\left|\eta \int_{\gamma}^{\alpha} \exp (i \phi(z)) d z\right| .
$$

It therefore suffices to show that

$$
\left|\eta \int_{\gamma}^{\alpha} \exp (i \phi(z)) d z\right| \leq C
$$

$$
\begin{aligned}
& \text { Now } \phi^{\prime}(z)=\xi r^{\prime}(z)+\eta+\tau / z \text {, so for } z \text { in }[\gamma, \alpha], \\
& \qquad\left|\phi^{\prime}(z)\right| \geq|\eta|-|\xi| A / 3-|\eta| / 3 \geq|\eta| / 3 .
\end{aligned}
$$

Since $\left(z^{2} \phi^{\prime \prime}(z)\right)^{\prime}$ is of constant sign in $[\gamma, \alpha], z^{2} \phi^{\prime \prime}(z)$ and so also $\phi^{\prime \prime}(x)$ is zero for at most one value of $z$ in $[\gamma, \alpha]$ and $[\gamma, \alpha]$ splits into at most two intervals in which $\phi^{\prime}$ is monotone. Breaking the integral in (A.7) up into the integrals over these subintervals and applying van der Corput's lemma then proves (A.7).

\section{REFERENCES}

1. M. Cowling, On Littlewood-Paley-Stein theory, Rend. Circ. Mat. Palermo Suppl. 1 (1981), 21-55.

2. M. Cowling and G. Mauceri, Inequalities for some maximal functions. I, Trans. Amer. Math. Soc. 287 (1985), 431-455.

3. A. Greenleaf, Principal curvature and harmonic analysis, Indiana Math. J. 30 (1982), 519-537. 
4. C. D. Sogge and E. M. Stein, Averages of functions over hypersurfaces in $\mathbf{R}^{n}$, Invent. Math. 82 (1985), 543-556.

5. E. M. Stein, Maximal functions: spherical means, Proc. Nat. Acad. Sci. U.S.A. 73 (1976), 2174-2175.

6. __ Some problems in harmonic analysis, Proc. Sympos. Pure Math., vol. 35, part I, Amer. Math. Soc., Providence, R.I., 1979, pp. 3-20.

7. E. M. Stein and S. Wainger, Problems in harmonic analysis related to curvature, Bull. Amer. Math. Soc. 84 (1978), 1239-1295.

8. E. M. Stein and G. Weiss, Introduction to Fourier analysis on Euclidean spaces, Princeton Univ. Press, Princeton, N.J., 1971.

SCHOOL OF MATHEMATICS, UNIVERSity OF NEW SOUTH WALES, KeNSINGTON, NeW South Wales 2033, Australia

ISTITUTO Di MATEMATICA, UNIVERSitÀ Di GenOVA, I-16132 GenOVA, ITALY 